\title{
PRÁTICAS INFORMACIONAIS EM AMBIENTES VIRTUAIS
}

\author{
INFORMATION PRACTICES IN VIRTUAL \\ ENVIRONMENTS
}

\author{
Laelson Felipe da Silva ${ }^{a}$ \\ Wagner Junqueira de Araújob \\ Henry Poncio Cruz de Oliveirac \\ Edvaldo Carvalho Alves ${ }^{d}$
}

\begin{abstract}
RESUMO
Introdução: estuda a produção sobre a temática "Práticas Informacionais em Ambientes Virtuais", considerando que as relações entre as tecnologias da informação e comunicação evoluem e modificam as práticas dos sujeitos, o que demanda abordagens complexas nos ambientes informacionais e que envolvem o contexto social e cultural. Objetivo: identificar, no período de 2010 a 2019, trabalhos que abordam o tema "práticas informacionais em ambientes virtuais", analisando aspectos quantitativos bibliométricos e altmétrico, e qualitativos relacionados aos conceitos mais recorrentes utilizados em seus respectivos referenciais teóricos. Metodologia: revisão sistemática de literatura, por meio de consulta as bases de dados: Emerald Group Publishing Limited, Information Science ad Technology Abstracts, Library and Information Scicente Abstracts, Library, Information Science \& Technology Abstracts with Full Text, Scopus e Web of Science. Resultados: foram identificados e analisados 58 artigos pertinentes ao tema; nos quais a palavra-chave mais recorrente foi "letramento informacional". Os conceitos utilizados para fundamentação teórica dos textos abordam: "estudos de usuários", "resiliência informacional", letramento informacional", "práticas informacionais" e "ambientes virtuais"; os países com maior produção compreendem o Reino Unido e Estados Unidos da América; Given, L. M. aparece como autor com maior número de citações. Conclusões: a integração de estudos de práticas informacionais em ambientes virtuais ainda está em processo de consolidação e tem gradativamente expandido suas aplicações empíricas para ambientes virtuais e não somente aos ambientes físicos.
\end{abstract}

a Doutorando do Programa de Pós-Graduacão em Ciência da Informação da Universidade Federal da Paraíba (PPGCI-UFPB). E-mail: laelsonfelipesilva@gmail.com.

b Doutor em Ciência da Informação pela Universidade de Brasília (UnB). Docente do Programa de Pós-Graduação em Gestão nas Organizações Aprendentes da Universidade Federal da Paraíba (PPGOA-UFPB). E-mail: wagnerjunqueira.araujo@gmail.com.

c Doutor em Ciência da Informação pela Universidade Estadual Paulista (UNESP). Docente do Programa de Pós-Graduação em Ciência da Informação da Universidade Federal da Paraíba (PPGCI-UFPB). E-mail: henry.poncio@gmail.com.

d Doutor em Ciências Sociais pela Universidade Federal de São Carlos (UFSCar). Docente do Programa de Pós-Graduação em Ciência da Informação da Universidade Federal da Paraíba (PPGCl-UFPB). E-mail: edvaldocalves@gmail.com. 
Descritores: Práticas informacionais. Ambientes virtuais. Letramento Informacional. Resiliência informacional. Tecnologia da informação e comunicação.

\section{INTRODUÇÃO}

Percebe-se, desde sua origem, um alargamento do campo dos Estudos de Usuários a partir da necessidade de se analisar um objeto complexo como o fenômeno informacional em uma sociedade "permeada por matrizes de significações diferentes e conflitivas e ainda múltiplas, concorrentes ou superpostas" (MARTELETO, 1995, p. 7).

Pinto e Araújo (2012) afirmam que o conceito de práticas informacionais se insere no campo da Ciência da Informação $(\mathrm{Cl})$ com objetivo de proporcionar a compreensão de elementos complexos que compõem as relações dos sujeitos com a informação, de modo que proporciona abranger aspectos sociais e culturais dantes não considerados em estudos tradicionais.

Portanto, a adoção da terminologia práticas informacionais e da postura sociocultural para empreender estudos daquele que passamos a denominar não mais usuário da informação e sim sujeito informacional (ressaltando desse modo seu caráter de ator) é consistente com o momento histórico da valorização do contexto nas investigações, considerando as relações dialógicas entre sujeito e contexto (DUARTE; ARAÚJO; DE PAULA, 2017, p. 4).

Tendo em vista a conexão da $\mathrm{Cl}$ com os desafios contemporâneos e as dinâmicas socioculturais que envolvem práticas informacionais em ambientes virtuais (acessados cada vez mais por meio das tecnologias da informação e comunicação), o campo é central para a produção de conteúdos informacionais pautados neste contexto tecnológico.

Assim empreendemos esta revisão sistemática de literatura acerca do tema "Práticas informacionais em ambientes virtuais", a fim de responder o seguinte questionamento: como se apresenta a produção científica acerca das análises de práticas informacionais em ambientes virtuais?

Objetivamos identificar dentro do período de 2010 a 2019, quais trabalhos abordam a temática referida, identificando aspectos bibliométricos e altmétricos dessa produção, bem como os conceitos mais recorrentes utilizados em seus 
respectivos referenciais teóricos.

Desta maneira, o presente trabalho está estruturado de modo a apresentar o desenvolvimento do conceito de práticas informacionais no campo dos estudos de usuários, seguido do conceito de ambientes informacionais; os procedimentos metodológicos; os resultados obtidos; e as considerações finais.

\section{PRÁTICAS INFORMACIONAIS E AMBIENTES VIRTUAIS}

Na Ciência da Informação, mais especificamente no campo de estudos de usuários, tem havido um significativo desenvolvimento teórico que vem permitindo, ao longo de décadas, a inserção de conceitos oriundos de outras ciências com o intuito de melhor compreender a relação sujeito e informação (SILVA, 2019).

A estes estudos são associados conhecimentos oriundos por exemplo das Ciências Sociais, o que possibilita abordar os usuários da informação de maneira complexa, contextualizada, considerando instâncias múltiplas que envolvem tanto estruturas mentais quanto estruturas sociais e a interpretação que os sujeitos dão a estes elementos.

Neste sentido, o conceito de práticas informacionais é posto como um dos que fornece ferramentas, teórico-metodológicas, para conceber esta hermética relação que envolve sujeito, informação e sociedade (GONZÁLEZ-TERUEL, 2005; ARAúJO, 2016).

Todavia, para o pleno entendimento das práticas informacionais, Rocha, Grandra e Rocha (2017) salientam que se faz necessário compreender o conceito de práticas sociais, pois segundo as autoras, a compreensão de práticas informacionais só seria viabilizada com a análise do contexto social.

Não obstante, compreender prática, mesmo que seja um exercício complexo dadas suas inúmeras definições, também consiste em um elemento indispensável à compreensão de práticas informacionais.

Rocha, Grandra e Rocha (2017, p. 99), buscam condensar o conceito de prática a partir da seguinte colocação: "as teorias da prática rompem, portanto, tanto com a perspectiva objetivista nas ciências sociais e com o pensamento 
subjetivista presente nas abordagens do cognitivismo [...]". Marteleto (1995) trata práticas informacionais como um conceito que busca analisar as ações dos sujeitos em relação à informação, seja busca, acesso, uso ou disseminação, de maneira a considerar que estas ações ocorrem e são decorrentes das interações sociais, nas quais estão envolvidas instâncias semiológicas, que envolvem uma reação dialógica sujeito-contexto.

E neste sentido, as relações dos sujeitos com a informação se modificam à medida que fatores sociais, culturais e econômicos evoluem. Assim, o surgimento de novas técnicas e ferramentas explícitas nas tecnologias da informação e comunicação (TIC) são agentes catalisadores dessas transformações que influenciam no contexto educacional, profissional e pessoal (BELLONI, 2002).

Por conseguinte, ambientes virtuais apresentam-se como recursos utilizados para diversas finalidades que envolvem educação, disponibilização de acervos virtuais, dentre outros recursos informacionais. Tornam acessíveis ambientes com aparato informacional e educacional que fisicamente seriam restritos (BELLONI, 2002).

Consequentemente, outras questões surgem da interação dos usuários com os novos ambientes informacionais proporcionados pela proliferação de diferentes tecnologias, muitas vezes de manuseio complexo ou mesmo de acesso restrito, como é o caso de alguns ambientes virtuais (LORENZO; DZIUBAN, 2006).

Assim, analisar práticas informacionais em ambientes virtuais corresponde à busca de melhor compreensão de como sujeitos lidam com a informação nestes ambientes, de maneira a considerar também as intrincadas instâncias sociais que constroem sua percepção do mundo.

\section{PROCEDIMENTOS METODOLÓGICOS}

Quanto aos objetivos, a pesquisa se caracteriza como descritiva, visto que busca descrever características da produção científica que relacionam práticas informacionais em ambientes virtuais, como também aspectos inerentes aos 
seus(suas) respectivos(as) autores(as). A abordagem que o estudo assumiu o inclui nos estudos infométricos desenvolvidos na Ciência da Informação que evidenciam "redes e comunidades de pesquisa e suas relações estruturais, bem como a construção de mapas de visualização de literaturas" (ARAÚJO, 2018, p. 14).

Os termos de busca foram selecionados a partir de leituras prévias de trabalhos sobre práticas informacionais, considerando a área de aplicação de tais estudos (Ciência da Informação), sua ligação com outro ramo de estudos dentro deste campo científico (Estudos de Usuários) e os ambientes nos quais esperamos verificar a aplicabilidade destes (Ambientes Virtuais). Assim, os termos foram expressos na seguinte sentença de busca: "Information Practice" AND ("Information Science" OR "User studies" OR "Virtual Environments").

As bases de dados utilizadas para obtenção das referências foram: Emerald Group Publishing Limited (Emerald), Information Science and Technology Abstracts (ISTA), Library and Information Science Abstracts (LISA), Library, Information Science \& Technology Abstracts with Full Text (LISTA), Scopus (Elsevier) e Web of Science.

Consideramos um período de dez anos, 2010 a 2019. Refinamos a pesquisa de modo a utilizar apenas textos completos e revisados por especialistas, levamos em conta apenas artigos. Para subsidiar a Revisão Sistemática de Literatura (RSL) ${ }^{1}$.

$\mathrm{Na}$ busca empreendida nas bases de dados mencionadas recuperamos um total de 277 artigos; dentre esses, 14 estavam fora do período delimitado (2010-2019) e foram descartados, nos proporcionando um total parcial de 263. Em seguida, desses 263 eliminamos 68 textos duplicados, resultando em 195 elegíveis para próxima etapa.

Após a leitura dos resumos dos textos remanescentes, verificamos que 137 estudos não diziam respeito aos objetivos dessa revisão. $O$ resultado final obtido compreendeu um total de 58 publicações que subsidiaram nossas análises.

1 Utilizamos as orientações do Preferred Reporting Items for Systematic reviews and MetaAnalyses - PRISMA (MOHER et al., 2009). 
Figura 1 - Etapas da revisão.
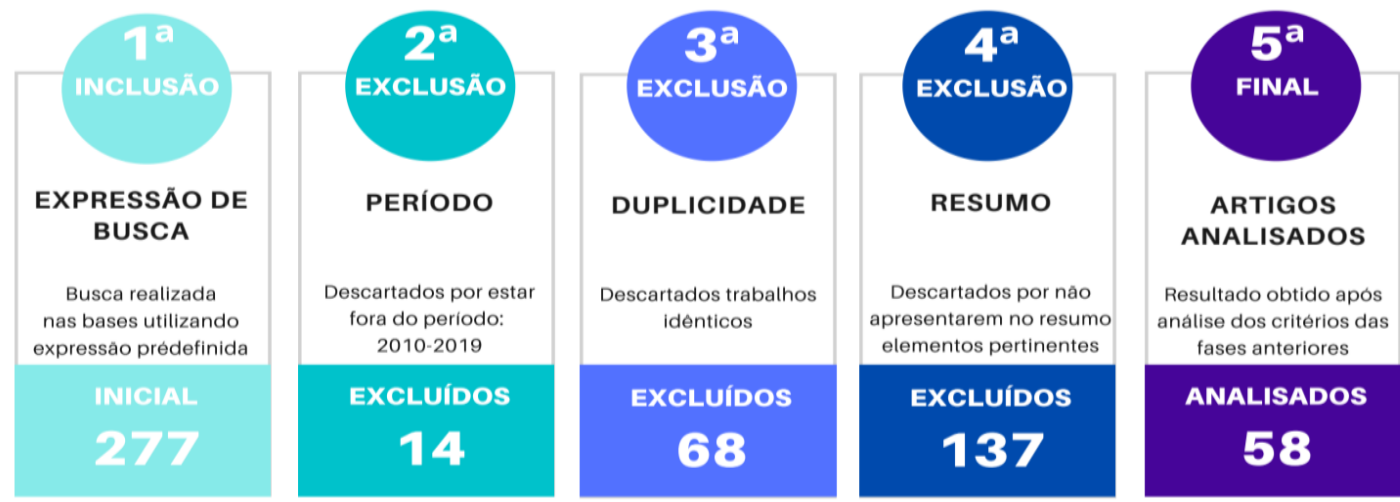

Bases de dados: Emerald Group Publishing Limited (Emerald), Information Science and Technology Abstracts (ISTA), Library and Information Science Abstracts (LISA), Library, Information Science \& Technology Abstracts with Full Text (LISTA), Scopus (Elsevier) e Web of Science;

Expressão de Busca: Information Practice" AND ("Information Science" OR "User studies" OR "Virtual Environments"); Período: 2010 a 2019.

Fonte: Dados da pesquisa (2019)

Todos os metadados dos artigos foram importados de suas bases para 0 software Zotero®, a partir do qual foi possível gerar os arquivos necessários às próximas etapas da revisão sistemática. O que compreendeu a utilização de softwares como Exce沼, para trabalhar os dados em planilhas e tabelas para exibição de resultados quantitativos; e Vosviewer $\AA^{\circledR}$ para gerar figuras de visualização das redes bibliométricas, de correlação entre as palavras-chave e de coautoria.

Foi utilizado o Nvivo® no processo de análise qualitativa, que permitiu verificar quais os conceitos mais recorrentes e preponderantes dos trabalhos analisados. Já para obtenção de aspectos altmétricos buscou-se dados na rede social científica Research Gate e no Google Citations.

\section{RESULTADOS E ANÁLISES}

Ao analisar as palavras-chave, mesmo que o tema pesquisado consista em "Práticas Informacionais em Ambientes Virtuais", outros termos indexadores assumiram significativa representatividade na composição das relações entre palavras-chave que os autores e autoras utilizaram para indexar seus trabalhos, como representado na figura 2 , que inclui também as correlações entre elas. 
Figura 2 - Palavras-chave mais frequentes

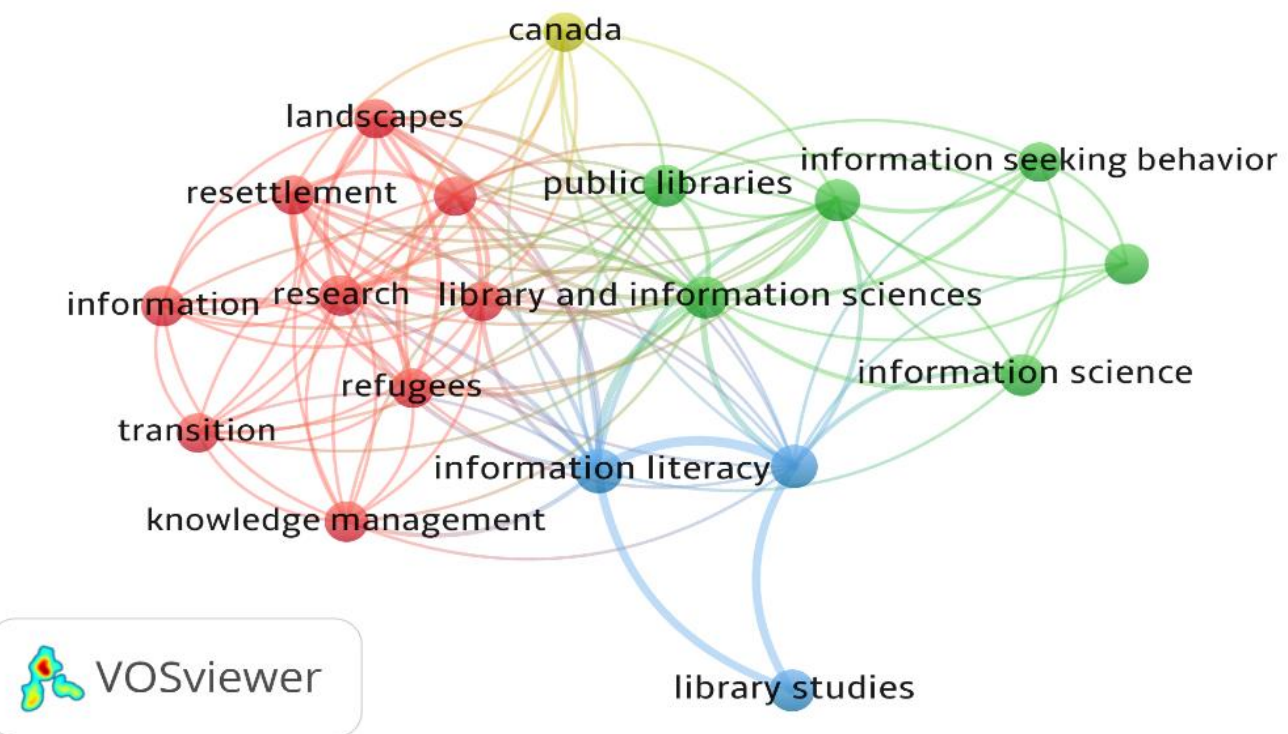

Fonte :Dados da pesquisa (2019)

Dentre os termos apresentados, "letramento informacional" (information literacy) é um dos mais recorrentes. A palavra-chave "Biblioteconomia e Ciência da Informação" (Library and Information Science), aparece como um termo que indica a área no qual se situam os estudos de práticas informacionais.

Os dados obtidos permitiram verificar também, dentre outros aspectos, quais os artigos mais relevantes a considerar o número de vezes que estes foram citados por outros autores. No quadro 1 são elencados os dez trabalhos mais citados dentre os analisados.

Quadro 1 - Títulos, periódicos e ano dos artigos mais citados

\begin{tabular}{|c|c|c|c|}
\hline Título do documento & Periódico & Ano & $\begin{array}{c}\mathbf{N}^{\circ} \text { de } \\
\text { citações }\end{array}$ \\
\hline $\begin{array}{c}\text { Framing information literacy as information } \\
\text { practice: site ontology and practice theory }\end{array}$ & $\begin{array}{c}\text { Journal of } \\
\text { Documentation }\end{array}$ & 2010 & 99 \\
\hline $\begin{array}{c}\text { Information behaviour of women: theoretical } \\
\text { perspectives on gender }\end{array}$ & $\begin{array}{c}\text { Journal of } \\
\text { Documentation }\end{array}$ & 2010 & 36 \\
\hline $\begin{array}{c}\text { Modelling information-seeking behaviour of } \\
\text { graduate students at Kuwait University }\end{array}$ & $\begin{array}{c}\text { Journal of } \\
\text { Documentation }\end{array}$ & 2012 & 35 \\
\hline $\begin{array}{c}\text { Supporting Informed Learners in the Twenty- } \\
\text { first Century }\end{array}$ & Library Trends & 2012 & 34 \\
\hline $\begin{array}{c}\text { The connection between purposive } \\
\text { information seeking and information } \\
\text { encountering: A study of Icelanders' health } \\
\text { and lifestyle information seeking }\end{array}$ & $\begin{array}{c}\text { Journal of } \\
\text { Documentation }\end{array}$ & 2010 \\
\hline
\end{tabular}




\begin{tabular}{|c|c|c|c|}
\hline $\begin{array}{c}\text { Agricultural information needs and sources of } \\
\text { the rural farmers in Tanzania: A case of Iringa } \\
\text { rural district }\end{array}$ & Library Review & 2013 & 34 \\
\hline $\begin{array}{l}\text { LIS research on information sharing activities } \\
\text { - people, places, or information }\end{array}$ & $\begin{array}{c}\text { Journal of } \\
\text { Documentation }\end{array}$ & 2012 & 31 \\
\hline $\begin{array}{l}\text { How poor informationally are the information } \\
\text { poor?: Evidence from an empirical study of } \\
\text { daily and regular information practices of } \\
\text { individuals }\end{array}$ & $\begin{array}{l}\text { Journal of } \\
\text { Documentation }\end{array}$ & 2010 & 21 \\
\hline $\begin{array}{c}\text { Stranger in a strange land; enabling } \\
\text { information resilience in resettlement } \\
\text { landscapes }\end{array}$ & $\begin{array}{l}\text { Journal of } \\
\text { Documentation }\end{array}$ & 2015 & 21 \\
\hline $\begin{array}{c}\text { Situating information literacy in the disciplines: } \\
\text { A practical and systematic approach for } \\
\text { academic librarians }\end{array}$ & $\begin{array}{l}\text { Reference } \\
\text { Services } \\
\text { Review }\end{array}$ & 2015 & 18 \\
\hline
\end{tabular}

Fonte: Dados da pesquisa (2019)

Para entender a composição teórica dos estudos, foram identificados, com auxílio do software Nvivo, em cada trabalho, os conceitos que os constituem; isso também nos possibilitou analisar a recorrência e a hierarquia desses conceitos.

Assim, à medida que determinado conceito foi identificado em um artigo, ele foi ligado a um "nó" e cada vez que este mesmo conceito se repetia nos demais artigos o "nó" foi sendo alimentado. Ao final desse processo, obtivemos os conceitos mais abrangentes.

Considerando o total de 58 trabalhos, a temática Estudos de Usuários ocorreu nos 58; Ambientes Virtuais em 50; Práticas Informacionais em 43 (mesmo que esse conceito, principal de nossa análise, não ocorre em todos os trabalhos, algumas abordagens de estudos de usuários, letramento, e resiliência representam abordagens muito próximas a ele); Letramento Informacional em 39, Resiliência Informacional em 34.

Dessa maneira, o quadro 2 apresenta o complexo panorama teóricoconceitual dos trabalhos, no qual estão conceitos ligados a origem e ao desenvolvimento do tema Práticas Informacionais. 


\section{Quadro 2 - Quadro conceitual}

\begin{tabular}{|c|c|}
\hline \multirow[b]{2}{*}{$\begin{array}{l}\text { Estudos de Usuários } \\
\text { (User Studies) }\end{array}$} & alés-Teruel (2018, p. 493) \\
\hline & $\begin{array}{l}\text { Os estudos de usuários definem como a indicação } \\
\text { sistemática das características, necessidades, condução e } \\
\text { opinião dos usuários (potenciais e reais) dos sistemas de } \\
\text { informação. }\end{array}$ \\
\hline \multirow[b]{2}{*}{$\begin{array}{c}\text { Ambientes virtuais } \\
\text { (Virtual } \\
\text { environments) }\end{array}$} & Schemil, Eppler e Freitas (2012, p. 838) \\
\hline & $\begin{array}{l}\text { Em geral, os ambientes virtuais tentam fornecer um ambiente } \\
\text { em que o usuário se sinta totalmente imerso e presente. A } \\
\text { imersão no sentido tradicional denota a tecnologia do } \\
\text { ambiente virtual e sua interface de usuário que, juntas, levam } \\
\text { a uma sensação de presença. }\end{array}$ \\
\hline \multirow[b]{2}{*}{$\begin{array}{c}\text { Práticas } \\
\text { informacionais } \\
\text { (Information practice) }\end{array}$} & Savolainen $(2007$, p. 126$)$ \\
\hline & $\begin{array}{l}\text { Esse conceito confere um papel central ao social e cultural, } \\
\text { fatores que qualificam a busca de informações e dedicam } \\
\text { atenção aos processos de compartilhamento de } \\
\text { informações. }\end{array}$ \\
\hline \multirow[b]{2}{*}{$\begin{array}{c}\text { Letramento } \\
\text { informacional } \\
\text { (Information Literacy) }\end{array}$} & Lloyd (2010, p 245) \\
\hline & $\begin{array}{l}\text { O letramento informacional é uma prática rica e complexa de } \\
\text { informação social construída de acordo com entendimentos } \\
\text { práticos específicos, regras e características teleoafetivas } \\
\text { que caracterizam um site ou configuração social. }\end{array}$ \\
\hline \multirow[b]{2}{*}{$\begin{array}{l}\text { Resiliência } \\
\text { informacional } \\
\text { (Information } \\
\text { Resilience) }\end{array}$} & Lloyd $(2015$, p. 12) \\
\hline & $\begin{array}{l}\text { O uso do termo resiliência informacional descreve o } \\
\text { resultado de práticas como o letramento informacional e } \\
\text { enfatiza o papel central que o acesso e o uso da informação } \\
\text { desempenham ao permitir pessoas a transição através de } \\
\text { tempos de incerteza. }\end{array}$ \\
\hline
\end{tabular}

Fonte: Dados da pesquisa (2019).

O conceito Estudos de Usuários trata dos estudos empreendidos em uma relevante área da Ciência da Informação. Os Estudos de Usuários têm possibilitado significativos avanços teóricos, implementando à Ciência da Informação conceitos oriundos de áreas diversas. Dentre estas áreas, a Sociologia forneceu abordagens e conceitos que posteriormente permitiram a constituição do campo dos Estudos de Práticas Informacionais (GONZALÉSTERUEL, 2018).

Os conceitos "Resiliência Informacional" e "Letramento Informacional" são apresentados nos textos como conceitos complementares. Isto se dá de forma tão intrínseca que para definir o conceito de Resiliência a autora utiliza do conceito de Letramento. Esses dois conceitos levam em consideração aspectos inerentes também ao conceito de Práticas Informacionais, pois começa a 
considerar os sujeitos informacionais de maneira contextualizada em suas práticas cotidianas, em suas práticas sociais.

O conceito Práticas Informacionais está atrelado ao conceito de práticas sociais, que antecedem as abordagens informacionais da $\mathrm{Cl}$. Para Savolainen (2007) toda prática que envolve a informação é uma prática social e "é por meio do estudo das práticas sociais ou culturais que são reveladas as práticas informacionais" (ROCHA; GANDRA; ROCHA, 2017, p. 99). Desta maneira, insere-se também a perspectiva da "práxis da informação" de Savolainen (2007).

Ambientes Virtuais é um conceito que, muitas vezes, está diluído nos trabalhos sem que seja possível verificar uma clara definição de suas propriedades e delimitações.

A definição apresentada no quadro anterior consiste na análise das referências utilizadas nos trabalhos e envolvem: ambientes digitais, ambientes virtuais, arquivo digital, bibliotecas virtuais, ensino a distância, ensino digital, ensino virtual, informação digital, realidade aumentada e realidade virtual.

O quadro conceitual reflete o desenvolvimento e consolidação dos Estudos de Práticas Informacionais que avança de análises empíricas em ambientes físicos, e posteriormente evolui ao ritmo das práticas dos sujeitos que se movem em direção aos ambientes virtuais, seja no sentido de interações recreativas, afetivas, profissionais e/ou educacionais.

No tocante aos países de origem, no Reino Unido foram identificados 13 artigos; dos Estados Unidos da América, 9; da Finlândia, 9; da Austrália, 5; do Canadá, 5; da Dinamarca, 3; da Suécia, 3; da África do Sul, 1; da Bélgica, 1; Brasil, 1; da China, 1; da Coreia do Sul, 1; da França, 1; da Islândia, 1; de Israel, 1; do México, 1; da Noruega, 1; da Nova Zelândia, 1; da Polônia, 1; da Tanzânia, 1; e da Espanha, também 1 artigo.

Os dados da origem da produção, nos mostram que são as universidades europeias que lideram com o maior número de publicações por instituição.

$\mathrm{Na}$ tabela 1 estão listadas as universidades que produziram mais de um trabalho sobre o tema. As demais universidades, com apenas um trabalho cada, estão condensadas no item "outras instituições. 
Tabela 1 - Produção por instituição

\begin{tabular}{ccc}
\hline Instituição & País & Trabalhos \\
\hline Universidade de Tempere & Finlândia & 5 \\
Universidade de Louisville & Estados Unidos & 3 \\
Universidade de Aberystwyth & Reino Unido & 2 \\
Universidade de Curtin & Austrália & 2 \\
Universidade de Robert Gordon & Reino Unido & 2 \\
Universidade de Borås & Suécia & 2 \\
Universidade da Califórnia em Angeles & Estados Unidos & 2 \\
Universidade de Strathclyde & Reino Unido & 2 \\
Universidade de Toronto & Canadá & 2 \\
Outras instituições & - & 36 \\
\hline Total & & 58 \\
\hline
\end{tabular}

Fonte: Dados da pesquisa (2019)

Na Finlândia, a Universidade de Tempere concentra cinco trabalhos cujos autores são Tuomas J. Harviaine; Amon Rapp; Pertti Vakkiri; Crystle Martin; Indunn Bøyum; Svanhild Aabø; Tumsifu Elly; Ephraen Epafra Silayo. Já, a segunda instituição em número de artigos publicados, a Universidade de Louisville, nos Estados Unidos, reúne três trabalhos cuja autoria é de responsabilidade de Yuanyuan Feng; Denise E. Agosto; Elysia Guzik; Konstantina Martzoukou; Simon Burnett.

Com base nos autores mais citados, foi elaborado o quadro 3 , no qual estão apresentados os cinco com mais citações. No topo lista, Annemaree Lloyd com 3.686 citações seguida de Reijo Savolainen com 3.226. Para construção deste quadro foram consideradas as citações dos trabalhos cujos temas são ligados ao conceito de práticas informacionais.

\section{Quadro 3 - Índices de citações para autores que mais publicaram}

\begin{tabular}{|ll|c|c|}
\hline & Autores(as) & Citações & Fonte \\
\hline 1) & Annemaree Lloyd & 3686 & Google Citations \\
\hline 2$)$ & Reijo Savolainen & 3226 & Google Citations \\
\hline 3$)$ & Christine Bruce & 2287 & Google Citations \\
\hline 4$)$ & David Bawden & 1832 & Google Citations \\
\hline 5) & Andrew M. Cox & 1606 & Google Citations \\
\hline
\end{tabular}

Fonte: Dados da pesquisa (2019)

Annemaree Lloyd e Reijo Savolainen são os autores responsáveis pelo desenvolvimento do conceito de práticas informacionais a partir da área da 
Ciência da Informação.

A figura 3, a seguir, mostra a rede de coautoria. Compreende as relações de produção entre os(as) autores(as). Todavia, mesmo que geograficamente a produção esteja concentrada em uma determinada região da Europa, tal proximidade não garante a construção de uma rede coesa e diversa de autores(as).

\section{Figura 3 - Relação de coautoria}

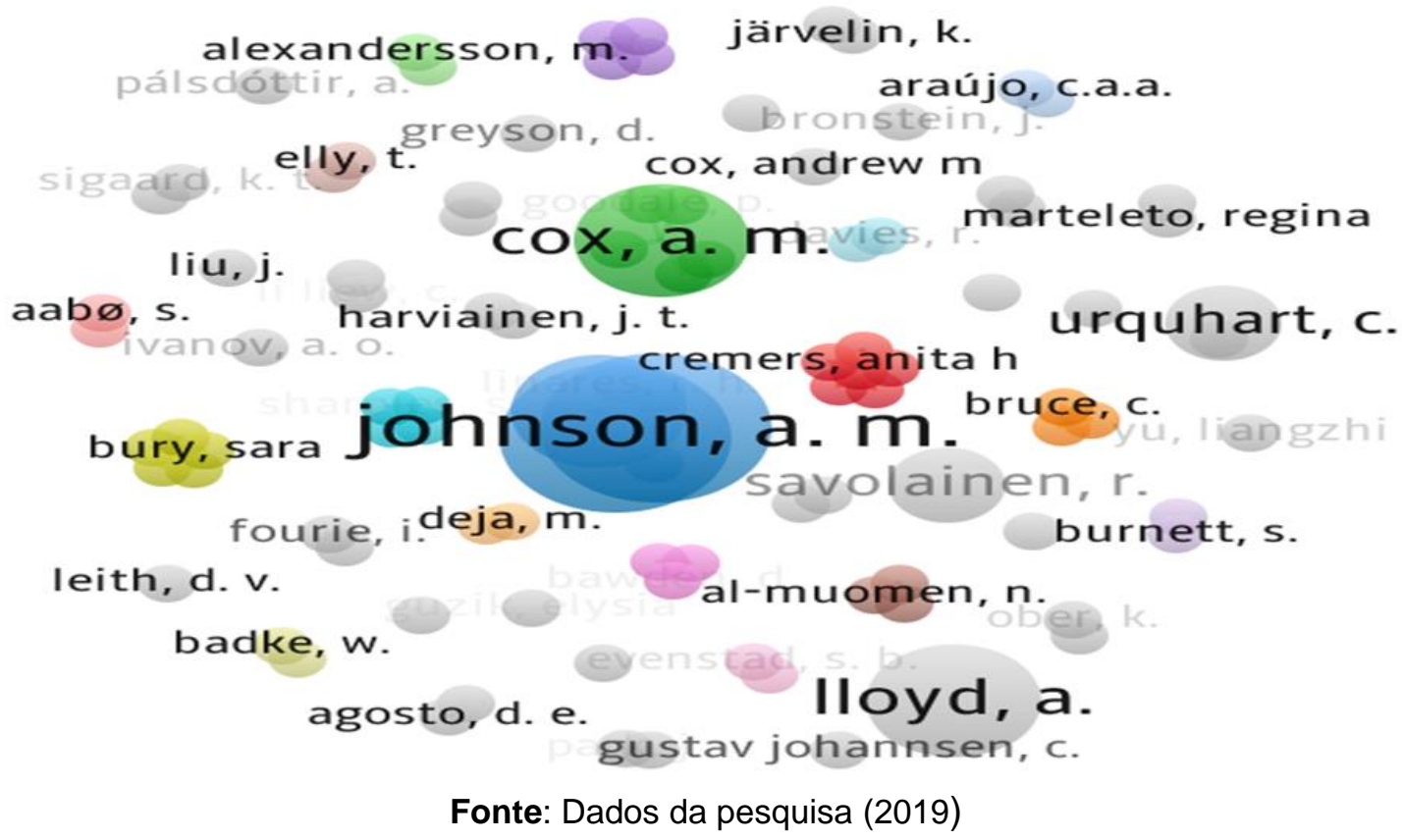

Foi identificado que os(as) principais autores(as) do tema produzem de maneira "isolada", sem que se articulem em uma rede complexa e expandida. As relações de coautoria que se mostraram expressivas, somente dentro de seus próprios grupos institucionais, elencados a seguir: Reijo Savolainen (2019, 2017); Christine Uequhart (2018); Andrew M. Cox (2018); Andrew M. Cox, Brian Griffin e Jenna Hartel (2017); Andrew M. Cox; Pamela Mckinney e Paula Goodale (2017); Annemaree Lloyd (2017, 2015, 2015); Dylan G. Schouten, Nanja J. Smets, Marianne Driessen, Knup Fuhri; Mark A. Neerincx, Anita H. Cremers (2017); Anna Marie Johnson, Claudene Sproles e Robert Detmering (2010); Christine Uequhart e Alison Yeoman (2010).

Foram extraídos da Research Gate os índices denominados de Research 
Score, Citations, Reads e Recomendations.

Ainda quanto aos autores e autoras, a rede social científica Score Research Gate (RG Score) mede, entre outros itens, a "reputação científica" com base em como o trabalho é recebido pelos colegas da comunidade, assim, a pontuação RG é calculada com base em diferentes contribuições que o pesquisador compartilha no ResearchGate ou adiciona ao seu perfil, como artigos publicados, projetos, perguntas e respostas, feedback, etc. (Research Gate, 2019, não paginado).

\section{Quadro 5 - Altimétrica dos(as) Autores(as)}

\begin{tabular}{|l|c|c|c|c|}
\hline Autores(as) & RG Score & Citation & Reads & Recommendation \\
\hline Bawden, David & 42,22 & 4043 & 12479 & 23 \\
\hline Savolainen, Reijo & 33,42 & 3410 & 12851 & 15 \\
\hline Farrell, Robert & 33,27 & 2345 & 9009 & 9 \\
\hline Urquhart, Christine & 31,56 & 2079 & 13389 & 84 \\
\hline Fourie, Ina & 31,39 & 583 & 7921 & 7 \\
\hline Vakkari, Pertti & 29,98 & 2936 & 8425 & 9 \\
\hline Cox, Andrew Martin & 27,33 & 1189 & 6352 & 4 \\
\hline Greyson, Devon & 25,95 & 799 & 6203 & 10 \\
\hline
\end{tabular}

Fonte: Research Gate (2019)

O indicador RG Score, é uma métrica específica da rede social científica Research Gate, representa pontuação geral do pesquisador relacionada aos itens mensurados na RG.

O índice Citations indica o número de citações verificados de um ou de um conjunto de artigos que o autor disponibilizou na plataforma.

Já o índice Reads, de acordo com o site, é contado sempre que alguém visualiza um resumo de publicação, clica em uma figura, exibe ou baixa o texto completo, ou clica em um projeto, ou faz um questionamento ao autor.

Tais indicadores demonstram a atividade acadêmica, a influência e permite a interação do pesquisador na rede de contatos com outros autores.

O Research Gate Score de maior impacto é do autor David Dawden, está ligado à Universidade de Londres, especificamente ao Centro de Ciência da Informação da instituição, suas habilidades e experiência estão relacionadas à biblioteconomia. 


\section{CONSIDERAÇÕES FINAIS}

Esta revisão sistemática de literatura permitiu verificar e analisar diferentes aspectos da produção sobre a temática "Práticas Informacionais em Ambientes Virtuais" cuja aplicabilidade vem se intensificando e se consolidando em estudos empreendidos no campo da Ciência da Informação.

Os Estudos de Práticas Informacionais se apresentam em contraponto e/ou complementaridade aos Estudos de Usuários que mesmo representando uma área profícua da Ciência da Informação, não incluem, a partir da perspectiva sociológica, aspectos inerentes ao contexto sociocultural dos sujeitos abordados em suas pesquisas.

Entretanto, apesar da proximidade com os tradicionais Estudos de Usuários explícita na produção analisada, outros pontos foram considerados e evidenciam, ainda nas palavras-chave, o desenvolvimento em direção ao conceito de Práticas Informacionais. Termos como "Letramento Informacional" e "Resiliência Informacional" remetem a conceitos análogos, que começam a considerar as relações contextualizadas dos usuários. Estes usuários, posteriormente, passam a ser denominados sujeitos informacionais devido à estruturação do conceito de práticas.

Neste sentido, a análise conceitual aponta para um arcabouço teóricoconceitual cuja coerência se firma na abordagem oriunda dos estudos tradicionais de usuários e seu desenvolvimento vai se tornando mais complexo ao ponto de inserir relações contextualizadas em ambientes distintos das clássicas unidades informacionais.

Assim, o conceito de Estudos de Usuários representa a origem, o ponto no qual se iniciam as pesquisas com sujeitos e que avança para inserção de conceitos oriundos de outras áreas em suas análises. O que, posteriormente, proporcionou o desenvolvimento de inúmeras abordagens, inclusive as empregadas nos Estudos de Práticas Informacionais.

Outros dois conceitos que, na produção em análise, mostram-se como conceitos complementares, e foram associados por alguns dos autores, são os conceitos de Letramento Informacional e Resiliência Informacionais. Os estudos 
empíricos nos quais estes conceitos foram identificados demonstram significativa relação com conceito de Práticas Informacionais, pois analisam sujeitos contextualizados em seu processo de aquisição de "habilidades informacionais" em ambientes diversos.

O conceito de Práticas Informacionais, que implica na inserção da questão sociocultural na análise da relação dos sujeitos com a informação, dentre outros aspectos, perpassa os trabalhos analisados, de maneira que em quaisquer campos empíricos está presente uma análise de sujeitos informacionais mediante a apreensão de suas relações sociais na interação com a informação e leva em consideração aspectos sociais culturais e históricos na constituição dos indivíduos.

Desta maneira, considerar que estes sujeitos também interagem com os artefatos tecnológicos produzidos na sociedade na qual se inserem consiste em uma concepção que vem ganhando espaço na literatura. $O$ que nos leva a considerar o conceito de Ambientes Virtuais, nos textos verificados, apresentase de maneira dispersa, sem que seja demonstrada uma definição exata, todavia as referências utilizadas pelos(as) autores(as) apontam para uma concepção de relações tecno-mediadas por aparatos como computadores e celulares.

Assim a aplicabilidade do conceito de Práticas Informacionais em Ambientes Virtuais mostra-se como uma abordagem em evolução, pois a análise dos textos selecionados evidencia poucos aspectos explicitamente ligados ao que poderíamos denominar de fato de um estudo de práticas informacionais, pois o que há, em alguns casos, é a utilização de outros conceitos como subsídio para abordar o comportamento informacional.

Significativa parte dos artigos ainda se detêm ao estudo de sujeitos informacionais em suas relações empreendidas em ambientes físicos. Todavia isto não chega a ser uma limitação, mas implica em um processo de expansão, que por vezes, pode se mostrar lento. Mas é um avanço na direção da ampliação e diversificação destes estudos.

Neste sentido, devemos considerar que os autores que têm se dedicado ao Estudo de Práticas Informacionais ainda compõem uma rede de coautoria esparsa, cujas ligações não representam grandes expansões institucionais e ou 
geográficas. Os autores produzem em seus restritos grupos sem se "conectarem" com outros sujeitos fora deste grupo.

Mesmo com esta aparente limitação da realização de estudos com claras definições de Ambientes Virtuais, e restrita rede de coautoria oriunda da centralidade da produção Europeia, os Estudos de Práticas Informacionais têm se consolidado na constituição de um corpus que permeia distintas realidades empíricas. Passam a constituir uma das áreas de estudos na Ciência da Informação, enriquecendo o campo com suas múltiplas possibilidades metodológicas oriundas das Ciências Sociais.

\section{REFERÊNCIAS}

ARAÚJO, Carlos Alberto Ávila. O que é ciência da informação. São Paulo: KMA, 2018.

ARAÚJO, Carlos Alberto Ávila. Estudos de usuários da informação: comparação entre estudos de uso, de comportamento e de práticas a partir de uma pesquisa empírica. Informação em Pauta, v. 1, n. 1, p. 61-78, 2016. Disponível em:. Acesso em: 28 fev. 2019.

BAWDEN, David. Everyday practices of documentation, and the influence of information science. Journal of Documentation v. 65, n. 5, p. jd.2009.27865eaa.001, 4 set. 2010. Disponível em: http://www.emeraldinsight.com/doi/10.1108/jd.2009.27865eaa.001. Acesso em: 24 ago. 2019.

BELLONI, Maria Luiza. Ensaio sobre a educação a distância no Brasil.

Educação \& sociedade, v. 23, n. 78, p. 117-142, 2002. BELLONI, Maria Luiza. Ensaio sobre a educação a distância no Brasil. Educação \& sociedade, v. 23, n. 78, p. 117-142, 2002. Disponível em:

http://www.scielo.br/scielo.php?pid=S010173302002000200008\&script=sci_arttext. Acesso em: 04 abr. 2020.

BØYUM, Idunn; AAB $\varnothing$, Svanhild. The information practices of Business PhD students. New Library World Oslo and Akershus University College of Applied Science. v. 116, n. 3/4, p. 187-200 , 9 mar. 2015. Disponível em: http://www.emeraldinsight.com/doi/10.1108/NLW-06-2014-0073. Acesso em: 24 ago. 2019.

COX, Andrew M. Embodied Knowledge and Sensory Information: Theoretical Roots and Inspirations. Library Trends The University of Sheffield. v. 66, n. 3, p. 223-238, 2018. Disponível em: 
https://search.proquest.com/docview/2157692966?accountid=149610. Acesso em: 24 ago. 2019.

COX, Andrew M.; GRIFFIN, Brian; HARTEL, Jenna. What everybody knows: embodied information in serious leisure. Journal of Documentation

University of Newcastle. v. 73, n. 3, p. 386-406, 8 maio 2017. Disponível em: http://www.emeraldinsight.com/doi/10.1108/JD-06-2016-0073. Acesso em: 24 ago. 2019.

COX, Andrew Martin; MCKINNEY, Pamela; GOODALE, Paula. Food logging: an information literacy perspective. Aslib Journal of Information Management v. 69, n. 2, p. 184-200, 20 mar. 2017. Disponível em:

http://www.emeraldinsight.com/doi/10.1108/AJIM-12-2016-0208. Acesso em: 24 ago. 2019.

DUARTE, Adriana Bogliolo Sirihal; ARAÚJO, Carlos Alberto Ávila; DE PAULA, Claudio Paixão Anastácio. Práticas Informacionais: desafios teóricos e empíricos de pesquisa. Informação em Pauta, v. 2, p. 111-135, 2017. Disponível em:

http://www.periodicos.ufc.br/informacaoempauta/article/view/20650. Acesso em: 15 maio 2018.

ELLY, Tumsifu; EPAFRA SILAYO, Ephraem. Agricultural information needs and sources of the rural farmers in Tanzania: A case of Iringa rural district. Library

Review University of Dar es Salaam. v. 62, n. 8/9, p. 547-566 , 25 nov. 2013. Disponível em: http://www.emeraldinsight.com/doi/10.1108/LR-01-2013-0009. Acesso em: 24 ago. 2019.

FARRELL, Robert; BADKE, William. Situating information literacy in the disciplines: A practical and systematic approach for academic librarians. Reference Services Review University of New York. v. 43, n. 2, p. 319-340, 8 jun. 2015. Disponível em: http://www.emeraldinsight.com/doi/10.1108/RSR11-2014-0052. Acesso em: 24 ago. 2019.

FENG, Yuanyuan; AGOSTO, Denise E. From health to performance: Amateur runners' personal health information management with activity tracking technology. Aslib Journal of Information Management Drexel University. v. 71, n. 2, p. 217-240, 18 mar. 2019. Disponível em: https://www.emeraldinsight.com/doi/10.1108/AJIM-07-2018-0170. Acesso em: 24 ago. 2019.

GONZÁLEZ-TERUEL, Aurora. Cuatro tradiciones en la investigacion del usuario: user studies, information behavoiur, information practice e information experience. AIB Studi Universitat de València. v. 58, n. 3, p. 439-439, set. 2018. Disponível em: http://search.ebscohost.com/login.aspx?direct=true \&db=izh\&AN=136795734\&la ng=pt-br\&site=ehost-live. Acesso em: 21 ago. 2018. 
GUZIK, Elysia. Information Sharing as Embodied Practice in a Context of Conversion to Islam. Library Trends university of waterloo. v. 66, n. 3, p. 351-370, 2018. Disponível em:

https://search.proquest.com/docview/2157673433?accountid=149610.

HARVIAINEN, J. Tuomas; RAPP, Amon. Multiplayer online role-playing as information retrieval and system use: an ethnographic study. Journal of Documentation University of Tampere. p. JD-07-2017-0100, 24 jan. 2018. Disponível em: http://www.emeraldinsight.com/doi/10.1108/JD-07-2017-0100. Acesso em: 24 ago. 2019

JOHNSON, Anna Marie; SPROLES, Claudene; DETMERING, Robert. Library instruction and information literacy 2009. Reference Services Review University of Louisville. v. 38, n. 4, p. 676-768, 16 nov. 2010. Disponível em: https://www.emeraldinsight.com/doi/10.1108/00907321011090809. Acesso em: 24 ago. 2019.

JOHNSON, Anna Marie; SPROLES, Claudene; REYNOLDS, Latisha. Library instruction and information literacy, 2008. Reference Services Review University of Louisville. v. 37, n. 4, p. 463-553, 13 nov. 2010. Disponível em: https://www.emeraldinsight.com/doi/10.1108/00907320911007056. Acesso em: 24 ago. 2019.

LLOYD, Annemaree. Framing information literacy as information practice: site ontology and practice theory. Journal of Documentation Charles Sturt University. v. 66, n. 2, p. 245-258, 9 mar. 2010. Disponível em: https://www.emeraldinsight.com/doi/10.1108/00220411011023643. Acesso em: 24 ago. 2019.

LLOYD, Annemaree. Researching fractured (information) landscapes. Journal of Documentation University of Boras. v. 73, n. 1, p. 35-47, 2017. Disponível em: https://search.proquest.com/docview/1850711182?accountid=149610.

LLOYD, Annemaree. Stranger in a strange land; enabling information resilience in resettlement landscapes. Journal of Documentation University College London. v. 71, n. 5, p. 1029-1042 , 2015. Disponível em: https://search.proquest.com/docview/2121471930?accountid=149610.

LORENZO, George; DZIUBAN, Charles. Ensuring the net generation is net savvy. Educause learning initiative, v. 2, n. 2, p. 1-19, 2006. Disponível em: http://www.understandingxyz.com/index_htm_files/Net\%20Gen\%20paper.pdf. Acesso em: 04 abr. 2020.

MAUNGWA, Tumelo; FOURIE, Ina. Competitive intelligence failures: An information behaviour lens to key intelligence and information needs. Aslib Journal of Information Management University of Pretoria. v. 70, n. 4, p. 367-389,16 jul. 2018. Disponível em: https://www.emeraldinsight.com/doi/10.1108/AJIM-01-2018-0018. Acesso em: 24 ago. 2019. 
MARTELETO, Regina Maria. Cultura informacional: construindo o objeto informação pelo emprego dos conceitos de imaginário, instituição e campo social. Ciência da informação, v. 24, n. 1, 1995. Disponível em: http://revista.ibict.br/ciinf/article/view/613. Acesso em: 28 fev. 2019.

MARTIN, Crystle. An information literacy perspective on learning and new media. On the Horizon University of Wisconsin-Madison. v. 19, n. 4, p. 268275, 27 set. 2011. Disponível em: http://www.emeraldinsight.com/doi/10.1108/10748121111179394. Acesso em: 24 ago. 2019.

MARTZOUKOU, Konstantina; BURNETT, Simon. Exploring the everyday life information needs and the socio-cultural adaptation barriers of Syrian refugees in Scotland. Journal Of Documentation Roberto Gordon University. v. 74, n. 5, p. 1104-1132,10 set. 2018. Disponível em: https://www.emeraldinsight.com/doi/10.1108/JD-10-2017-0142. Acesso em: 24 ago. 2019.

MOHER, D. et al. Preferred Reporting Items for Systematic Reviews and MetaAnalyses: The PRISMA Statement. PLOS Medicine, v. 6, n. 4, p. 1-6, 2009. Disponível em:

http://journals. plos.org/plosmedicine/article/file?id=10.1371/journal.pmed. 10000 97\&type=pri ntable. Acesso em: 14 jun. 2018.

PINTO, Flávia Virgínia Melo; ARAÚJJO, Carlos Alberto Ávila. Contribuição ao campo de usuários da informação: em busca dos paradoxos das práticas informacionais. Transinformação, v. 24, n. 3, p. 219-226, 2012. Disponível em: https://www.redalyc.org/pdf/3843/384334891006.pdf. Acesso em: 23 abr. 2020.

RESEARCHGATE. Página inicial. 2019. Disponível em: https://www.researchgate.net. Acesso em: 21 dez. 2020

ROCHA, Eliane Cristina de Freitas; GANDRA, Tatiane Krempser; ROCHA, Janicy Aparecida Pereira. Práticas informacionais: nova abordagem para os estudos de usuários da informação. Biblios, n. 68, p. 96-109, 2017. Disponível em: http://www.scielo.org.pe/scielo.php?pid=S1562-

47302017000300007\&script=sci_arttext\&tIng=en. Acesso em: 23 abr. 2020.

SAVOLAINEN, Reijo. Information need as trigger and driver of information seeking: a conceptual analysis. Aslib Journal of Information Management Tempere University. v. 69, n. 1, p. 2-21, 16 jan. 2017. Disponível em: http://www.emeraldinsight.com/doi/10.1108/AJIM-08-2016-0139. Acesso em: 24 ago. 2019.

SAVOLAINEN, Reijo. Information behavior and information practice: reviewing the "umbrella concepts" of information-seeking studies. The Library Quarterly, v. 77, n. 2, p. 109-132, 2007. Disponível em: https://www.journals.uchicago.edu/doi/abs/10.1086/517840. Acesso em: 27 ago. 2019. 
SCHMEIL, Andreas; EPPLER, Martin J.; DE FREITAS, Sara. A structured approach for designing collaboration experiences for virtual worlds. Journal of the Association for Information Systems, v. 13, n. 10, p. 2, 2012. Disponível em: https://aisel.aisnet.org/jais/vol13/iss10/2/. Acesso em 13 set. 2019.

SCHOUTEN, Dylan G; M. et al. Requirements for a virtual environment to support the social participation education of low-literates. Universal Access in the Information Society, [S. I.], v. 16, n. 3, p. 681-698, 2017. Disponível em: https://doi.org/10.1007/s10209-016-0502-z. Acesso em: 23 abr. 2020.

SILVA, Laelson Felipe da et al. Práticas informacionais: LGBTQI+ e empoderamento no espaço LGBT. 2019. Disnponível em: https://repositorio.ufpb.br/jspui/bitstream/123456789/16342/1/Arquivototal.pdf. Acesso em: 23 abr. 2020.

URQUHART, Christine. Principles and practice in impact assessment for academic libraries. Information and Learning Science, [S. I.], v. 119, n. 1/2, UK, p. 121-134, 2018. Disponível em: https://doi.org/10.1108/LS-06-20170053. Acesso em: 23 abr. 2020. Acesso em: 23 abr. 2020.

URQUHART, Christine; YEOMAN, Alison. Information behaviour of women: theoretical perspectives on gender. Journal of Documentation, [S. I.], v. 66, n. 1, UK, p. 113-139, 2010. Disponível em:

https://doi.org/10.1108/00220411011016399. Acesso em: 23 abr. 2020.

VAKKARI, Pertti. Internet use increases the odds of using the public library. Journal of Documentation v. 68, n. 5, p. 618-638, 31 ago. 2012. Disponível em: https://www.emeraldinsight.com/doi/10.1108/00220411211256003. Acesso em: 24 ago. 2019.

\title{
INFORMATION PRACTICES IN VIRTUAL ENVIRONMENTS
}

\begin{abstract}
Introduction: it studies the production on the theme "Informational Practices in Virtual Environments", considering that the relations between information and communication technologies evolve and modify the practices of the subjects which demands complex approaches in informational environments and which include the social and cultural context. Objective: to identify, in the period from 2010 to 2019, papers that discuss the theme "Informational Practices in Virtual Environments", analyzing quantitative bibliometric and altimetric aspects of this production and qualitative aspects related to the most recurrent concepts used in their respective theoretical references. Methodology: systematic literature review, by consulting the databases: Emerald Group Publishing Limited, Information Science ad Technology Abstracts, Library and Information Scicente Abstracts, Library, Information Science \& Technology Abstracts with Full Text, Scopus and Web of Science. Results: 58 papers relevant to the topic were identified and analysed; in which the most frequently used keyword was
\end{abstract}


"information literacy". The concepts used for the theoretical basis of the texts are: "user studies", "informational resilience", "informational literacy", "informational practices" and "virtual environments"; the countries with the highest production include the United Kingdom and the United States of America; Given, L. M. is listed as the author with the highest number of citations. Conclusions: the integration of studies of informational practices in virtual environments is still in the process of consolidation and has gradually expanded its empirical applications to virtual environments and not only to physical environments.

Keywords: Information practices. Virtual environments. Information Literacy. Information resilience. Information and communication technologies.

\title{
PRÁCTICAS DE INFORMACIÓN EN ENTORNOS VIRTUALES
}

\begin{abstract}
RESUMEN
Introducción: Se estudia la producción sobre el tema "Prácticas informativas en entornos virtuales", considerando que las relaciones entre las tecnologías de la información y la comunicación evolucionan y modifican las prácticas de los sujetos, lo que exige enfoques complejos en los entornos informativos y que implican el contexto social y cultural. Objetivo: identificar, en el período de 2010 a 2019, obras que aborden el tema "Prácticas de Información en Ambientes Virtuales", analizando aspectos bibliométricos y altimétricos cuantitativos de esta producción y aspectos cualitativos relacionados con los conceptos más recurrentes utilizados en sus respectivas referencias teóricas. Metodología: revisión sistemática de la literatura, consultando las bases de datos: Emerald Group Publishing Limited, Information Science ad Technology Abstracts, Library and Information Scicente Abstracts, Library, Information Science \& Technology Abstracts with Full Text, Scopus y Web of Science. Resultados: Se identificaron y analizaron 58 artículos pertinentes al tema, en los que la palabra clave más recurrente fue "conocimientos básicos de información". Los conceptos utilizados para la base teórica de los textos son: "estudios de usuarios", "resiliencia informacional", "conocimientos básicos de información", "prácticas de información" y "entornos virtuales"; los países con mayor producción son el Reino Unido y los Estados Unidos de América; Given, L. M. aparece como el autor con el mayor número de citas. Conclusiones: la integración de los estudios de las prácticas informativas en los entornos virtuales se encuentra todavía en proceso de consolidación y ha ampliado gradualmente sus aplicaciones empíricas a los entornos virtuales y no sólo a los entornos físicos.
\end{abstract}

Descriptores: Prácticas informativas. Entornos virtuales. Alfabetización informacional. Resiliencia informacional. Tecnologí

Recebido em: 04.05 .2020

Aceito em: 26.10 .2020 